\title{
Using Conditional Random Fields for Decision-Theoretic Planning
}

\author{
Paul A. Ardis and Christopher M. Brown \\ ardis,brown@cs.rochester.edu \\ Dept. of Comp. Science, Univ. of Rochester \\ P.O. Box 270226, 734 Comp. Studies Bldg. \\ Rochester, NY 14627-0226
}

\begin{abstract}
We propose a means of extending Conditional Random Field modeling to decision-theoretic planning where valuation is dependent upon fullyobservable factors. Representation is discussed, and a comparison with existing decision problem methodologies is presented. Included are exact and inexact message passing schemes for policy making, examples of decision making in practice, extensions to solving general decision problems, and suggestions for future use.
\end{abstract}

\section{Introduction}

Although there exist various approaches to utility calculation of a decision sequence, we desire a means of describing a series of potentially-inhomogenous decisions that are fully, partially-, or un-ordered, along with an efficient means of computing and comparing decision valuations. Traditional formulations are inadequate, as the introduction of decision reordering results in a blowup in graph complexity and size. We have devised a means of applying traditional discriminative graphical model inference methods to the task of compact exact and approximate planning under full observation, and extend it to the generation of optimal strategies for general decision problems.

For the purposes of comparing our model to existing generative decision formulations, we will make use of Shenoy's Valuation Network (VN) [Shenoy, 2000] approach as it is the most intuitively similar means of modeling potentiallyasymmetric decision problems ("scenarios" in equivalent terminology), using complimentary graphical and numerical representations most similar to our Conditional Random Field (CRF) [Lafferty and Pereira, 2001] approach. Additionally, we will compare our approach with the more general Markov Logic Network (MLN) formalism [Richardson and Domingos, 2006] in its use of absolute valuations for hard constraints, although we prefer the CRF description for compactness (see Figure 1 and later discussion in Section 3). Other decision problem formulations such as decision trees, asymmetric Influence Diagrams (IDs) [Jensen and Vomlelova, 2002], and Sequential Valuation Networks (SVNs) [Demirer and Shenoy, 2006] will briefly appear as they relate to relative strengths or weaknesses of our approach. Additional general motivation may be found in planning for large Markov Decision Processes (MDPs) [Kearns et al., 1999], although this formulation does not suit our purposes without modifying a number of limiting factors (e.g., first-order Markov assumption, temporally-universal decision space, additive-only valuation) that compromise expressibility or compactness.

In short, our approach is to generate a CRF from the full problem description, producing a discriminative graphical model for policy making that we have dubbed a Discriminative Action-Reward Network (DARN). Decision problems are easily expressed using this framework, and instancespecific inference is based upon an efficient utilization of Loopy Belief Propagation (BP) [Pearl, 1988] using message passing [Yedidia et al., 2001]. Finally, optimal strategies for the general problem can be found by including additional instance likelihood factors and selecting the set of variable assignments with highest expected utility over all instances.

The remainder of this document is structured as follows: we define key terms in Section 2, explain the details of the DARN model and its inference in Sections 3 and 4, present modifications for general decision problem solving in Section 5 , and conclude in Section 6.

\section{Definitions}

To begin, we define a generic terminology for discussion. A decision problem is defined as an ordered tuple $(\mathbf{D}, \mathbf{C}, \mathbf{A}, \mathbf{O}, u(\cdot))$, where:

- $\mathbf{D}$ is a set of finite domains for $m$ variables

- $\mathbf{C}$ is a probability distribution for the simultaneous assignment of values of $n$ variables

- $\mathbf{A}$ is an alphabet of $m$ unique symbols

- $\mathbf{O}$ is a set of $m$ ! or fewer unique ordered tuples of $m$ unique elements of $\mathbf{A}$

- $u(\cdot)$ is a function whose domain is the Cartesian product of $\mathbf{O}$ and the $m$ and $n$ variable domains, and whose range is a set where at least one computable binary relation defines a strict total order

Specifically, D corresponds to all possible options ("alternatives" in equivalent terminology) for $m$ decisions, $\mathbf{C}$ corresponds to the influence of outside factors in the form of $n$ 


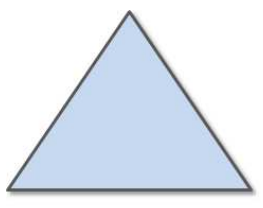

(a)

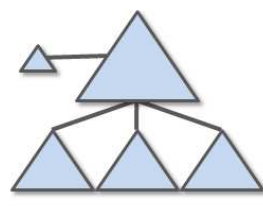

(b)

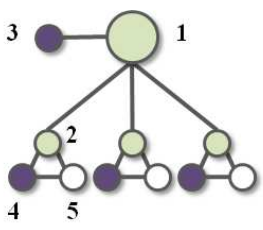

(c)

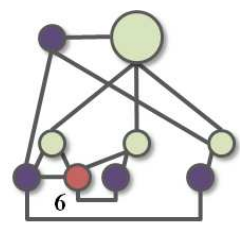

(d)

Figure 1: General Graph Construction for a Decision Problem: (a) Judging potential solutions is initially viewed as a single complex operation. (b) However, it is possible to describe a solution as a series of solutions to subproblems plus a temporal ordering. To judge an overall solution, then, is to judge these results together. (c) More specifically, a solution can be judged by a numerical score, where this score depends upon the benefit from each disparate decision. The final score (1) is computed from the decision scores (2) and the ordering (3), while the decision scores depend upon the decision selected (4) and other relevant factors (5), including decision order and outside influences. The decision may be constrained by the same factors, preventing a setting of impossible decisions. (d) Concretely, we can name the variables (6), decisions, and orderings that influence each score, expanding out the factors and removing duplicates. All data needed to compute an overall scoring are known, so it is possible to compute and compare these scores. A model should therefore be complete (represent all data points using graph nodes), correct (represent all data point connections using edges), clear (represent unique data points and connections using one or more disparate nodes or edges), and compact (contain a minimal number of nodes and edges). We suggest that the DARN model is all four, while earlier models and other graphical formulations are not clear or compact for problems where ordering is not fixed.

chance variables (independent of the $m$ decisions), A corresponds to a unique labeling of the $m$ decisions, $\mathbf{O}$ corresponds to allowed orderings of the $m$ decisions (listing their labels in their temporal order), and $u(\cdot)$ corresponds to the scoring of the $m$ decisions given the $n$ chance variables and the order of decisions made (minimally evaluating for unallowed decisions). That is, when faced with a series of decisions to make (in one or more allowed orders), it is possible to compute the relative value of the outcome and therefore determine what decisions guarantee a highly-valued result.

A strategy $\mathbf{S}$ for a decision problem is a set of variable assignments from $\mathbf{D}$ along an ordering appearing in $\mathbf{O}$. Incorporating the influence of $\mathbf{C}$, it is possible to determine an expected utility corresponding to each strategy, where this value is a member of the range of $u(\cdot)$ and is equal to an occurrence-weighted average of the values of $u(\cdot)$ produced using the strategy over an infinite timespan. When using realvalued numbers for the range of $u(\cdot)$, this expected utility is defined as $\int p(u(\cdot)=x \mid S) x d x$, although other formulations are used when the range of $u(\cdot)$ is otherwise defined. An optimal strategy is a strategy whose expected utility is maximally ordered in relation to the expected utility of all other strategies. In other words, an optimal strategy is the set of decisions expected to perform the best over time.

When solving a decision problem, the goal is to produce one or more optimal strategies. However, this may not be possible unless $\mathbf{D}, \mathbf{C}, \mathbf{O}$ and $u(\cdot)$ are all known during policy making (i.e., reasoning performed to produce a strategy). In many applications, in fact, $\mathbf{C}$ is not known and may not even be well-approximated by a small number of samples. Rather than attempting to determine a setting and ordering of decisions that is expected to do well over all $\mathbf{C}$, it is often a useful task to instead perform policy making on specific decision instances, where $\mathbf{C}$ is replaced by a set of unary spike distributions whose impulses are defined by a single joint sample from the original C. In practical terms, this corresponds to the selection of decisions that will perform well under a given setting of outside factors; we refer to this as decisiontheoretic planning. We describe a method for performing fast exact and approximate reasoning in that case, then demonstrate how it can be extended to solve full decision problems.

\section{The DARN Model}

The DARN model of a decision problem is specified at two levels - graphical and numerical. The numerical and graphical specifications of the DARN model together form a CRF (expressed as a factor graph [Kschischang et al., 2001]) where $p(Y \mid X)=u(\cdot)$; we will not provide a full introduction to CRF modeling, but instead instruct the reader to review the definitions and examples presented by [Sutton et al., 2007] if unfamiliar. The strength of this approach depends upon a factorization of $u(\cdot)$ such that $u(\cdot)=$ $h\left(g_{1}(\cdot), g_{2}(\cdot), \ldots, g_{m}(\cdot), O\right)$, where $g_{i}(\cdot)$ is the value gained (or lost) by the $i$ th decision, $O$ is an ordering with $O \in \mathbf{O}$, and $h(\cdot)$ is a function that unifies these terms to reflect their impact upon the final utility valuation. This approach allows for a larger domain of representable problems than traditional approaches (as final valuation is not restricted to being purely additive or multiplicative, and as decisions may be fully-, partially-, or un-ordered by modifying $h(\cdot)$ )

The details of the CRF structure are as follows:

- $Y$ consists of

- $y_{1}^{d e c}, y_{2}^{d e c}, \ldots, y_{m}^{d e c}$ whose domains form $\mathbf{D}$

- $y_{i}^{v a l}$ to record each of the $g_{i}(\cdot)$ factors of $u(\cdot)$

- $y_{i}^{\text {ord }}$ to record the value of $\mathrm{O}$

- $X$ consists of

- The $n$ observed variables $x_{1}, x_{2}, \ldots, x_{n}$ whose joint probability distribution forms $\mathbf{C}$

- The set of features ( $F$, as factor nodes) consists of 
- $f_{i}^{d e c}\left(Y_{i}^{d e c}, Y, X\right)$, whose parameters are $Y_{i}^{d e c}$ and the subset of $X \cup Y$ that determines if a decision is "allowed" (i.e., feasible given previous actions, environmental constraints, and decision ordering) according to the problem. Output is 0 if the decision is allowed, otherwise $-\infty$.

- $f_{i}^{v a l}\left(Y_{i}^{\text {dec }}, Y_{i}^{v a l}, Y, X\right)$, whose parameters are $Y_{i}^{\text {dec }}, Y_{i}^{v a l}$, and the subset of $X \cup Y$ that form the parameters of $g_{i}(\cdot)$ (i.e., variables influencing the value of the $i$ th decision). Output is 0 if $Y_{i}^{v a l}=$ $g_{i}(\cdot)$, otherwise $-\infty$.

- $f^{\text {tot }}\left(Y_{1}^{v a l}, Y_{2}^{\text {val }}, \ldots, Y_{m}^{v a l}, Y_{1}^{\text {ord }}, Y_{2}^{\text {ord }}, \ldots, Y_{m}^{\text {ord }}\right)$, whose parameters are the $Y$ variable assignments (i.e., factors of the joint utility) and the decision ordering. Output is $\ln (h(\cdot))$ if the ordering is allowed, otherwise $-\infty$.

As a result, an optimal strategy may be found by maximum-likelihood inference for $Y$ (explained in Section 4). Factor weights are not used, although replacing $f^{\text {tot }}(\cdot)=$ $\ln (h(\cdot))$ with $f^{t o t}(\cdot)=h(\cdot)$ or normalizing is allowed if valuations are only used in order-based comparison; other DARN uses (e.g., remorse quantification with approximate inference) will void their use.

We present an example for illustration, where the graphical level is shown in Figure 2. In this example, we are tasked with creating the ultimate sandwich. The available ingredients are: chicken, ham, cheese (Swiss and American), lettuce, tomato, butter, olive oil, and vinegar. Other than the last three, ingredients come in $100 \mathrm{~g}$ increments up to $500 \mathrm{~g}$; the final three are used in negligible quantity and are required. Scoring depends upon the ingredients as well as the order of their placement. Total weight is limited to $1 \mathrm{~kg}$, including a $200 \mathrm{~g}$ bun.

The rules that determine sandwich quality are as follows:

1. Butter is the key to a good sandwich: its appearance as the first ingredient solidifies its flavor impact (1 point).

2. Chicken is tasty ( 2 points per $100 \mathrm{~g}$ ), but ham is delicious (3 points per $100 \mathrm{~g}$ ).

3. Swiss cheese works well if there is an equal or greater amount of ham to pair it ( 2 points per $100 \mathrm{~g}$ ), but overpowers otherwise ( -1 points per $100 \mathrm{~g}$ unmatched).

4. American cheese is simple and tastes fine no matter what else is present ( 1 point per $100 \mathrm{~g}$ ).

5. Oil and vinegar will lessen the sandwich's impact if not placed immediately adjacent ( -4 points).

6. Tomato is yummy ( 2 point per $100 \mathrm{~g}$ ), as is lettuce ( 2 point per $100 \mathrm{~g}$ ), but both should be used sparingly (resulting in no additional points beyond $100 \mathrm{~g}$ ).

7. Be careful about bad tomatoes (there is a $50 \%$ chance of -1 point if tomato is included).

The details of the numerical level (showing ingredient names in place of $i$ for clarity) are below:

- $Y_{\text {butter }}^{\text {dec }}, Y_{\text {oil }}^{\text {dec }}, Y_{\text {vinegar }}^{\text {dec }} \in\{0\}$

- $Y_{i}^{\text {dec }} \in\{0,1,2,3,4,5\}$ for all other $i$

- $g_{\text {chicken }}\left(Y_{\text {chicken }}^{\text {dec }}\right)=2 * Y_{\text {chicken }}^{\text {dec }}$
- $g_{\text {ham }}\left(Y_{\text {ham }}^{\text {dec }}\right)=3 * Y_{\text {ham }}^{\text {dec }}$

- $g_{\text {swiss }}\left(Y_{\text {swiss }}^{\text {dec }}, Y_{\text {ham }}^{\text {dec }}\right)=2 * Y_{\text {swiss }}^{\text {dec }}$ if $Y_{\text {swiss }}^{\text {dec }} \leq Y_{\text {ham }}^{\text {dec }}$, otherwise $2 * Y_{\text {ham }}^{\text {dec }}-\left(Y_{\text {swiss }}^{\text {dec }}-Y_{\text {ham }}^{\text {dec }}\right)$

- $g_{\text {american }}\left(Y_{a m e r i c a n}^{\text {dec }}\right)=Y_{a m e r i c a n}^{\text {dec }}$

- $g_{\text {lettuce }}\left(Y_{\text {lettuce }}^{\text {dec }}\right)=2$ if $Y_{\text {lettuce }}^{\text {dec }}>0$, otherwise 0

- $g_{\text {tomato }}\left(Y_{\text {tomato }}^{\text {dec }}\right)=2$ if $Y_{\text {tomato }}^{\text {dec }}>0$ and $X_{\text {tomato }}=0$, 1 if $Y_{\text {tomato }}^{\text {dec }}>0$ and $X_{\text {tomato }}=1$, otherwise 0

- $g_{\text {butter }}\left(Y_{\text {butter }}^{\text {dec }}\right)=g_{\text {oil }}\left(Y_{\text {oil }}^{\text {dec }}\right)=g_{\text {vinegar }}\left(Y_{\text {vinegar }}^{\text {dec }}\right)=$ 0

- $f_{i}^{v a l}\left(Y_{i}^{v a l}, Y_{i}^{\text {dec }}, \cdot\right)=0$ if $Y_{i}^{v a l}=g_{i}(\cdot)$, otherwise $-\infty$

- $f_{i}^{d e c}(Y)=0$ if $\sum_{i} Y_{i}^{\text {dec }} \leq 8$, otherwise $-\infty$

- $h(\cdot)=\sum_{i} Y_{i}^{\text {val }}$ if $Y_{i}^{\text {ord }}$ unique, $-\infty$ otherwise (add 1 if $Y_{\text {butter }}^{\text {ord }}=1$ ) (subtract 4 if $\left|Y_{\text {oil }}^{\text {ord }}-Y_{\text {vinegar }}^{\text {ord }}\right|>1$ )

- $f^{t o t}=\ln (h(\cdot))$

One optimal choice of sandwich ingredients and order, then, is: butter, $500 \mathrm{~g}$ ham, $300 \mathrm{~g}$ Swiss cheese, $0 \mathrm{~g}$ of the rest, olive oil, vinegar. Assuming that the tomatoes are not bad, another optimal sandwich replaces $100 \mathrm{~g}$ of Swiss cheese with $100 \mathrm{~g}$ of tomatoes; in Section 4, we will show how to calculate these arrangements.

As a sidenote, we will consider how this problem would be expressed using existing decision-theoretic constructs. A decision tree would need branches for all 362880 feasible orderings. Likewise, an ID, VN, or SVN would require linear node growth in the number of orderings, as non-loopy paths are required by their various fusion algorithms [Shenoy, 2000; Demirer and Shenoy, 2006]. Similarly, an MDP would require a state space that encoded all previous actions (to restrict the next decision to remaining ingredients). In all of these cases, the result is a graph that is incomprehensibly large for human purposes. The undirected orderable approach taken by our model is therefore a serious advantage, as it allows for compact representation where decision structure has not changed beyond reordering, and where each decision has a separate decision space. The next step is to show how to use this representation for planning.

\section{Inference}

Using existing approaches for CRFs, inference in the DARN model is performed by Loopy BP via message passing. Although a number of optimizations are effective in reducing message density for general CRFs (e.g., Sparse Belief Propagation, a generalization of linear-chain Sparse ForwardBackward [Pal et al., 2006]), sparsification is not feasible for the DARN model without compromising the encoding of hard decision constraints ${ }^{1}$ Instead, we propose two customized message ordering schemes designed to reduce the number of required factor function computations.

\footnotetext{
${ }^{1}$ Since the exponentiation of $F \backslash f^{t o t}$ results in a constant value for allowed and zero for disallowed assignments, the removal of message entries is arbitrary and may disregard high-valuation assignments. This argument also rules out Sparse Mean Field approximation [Weinman et al., 2008].
} 


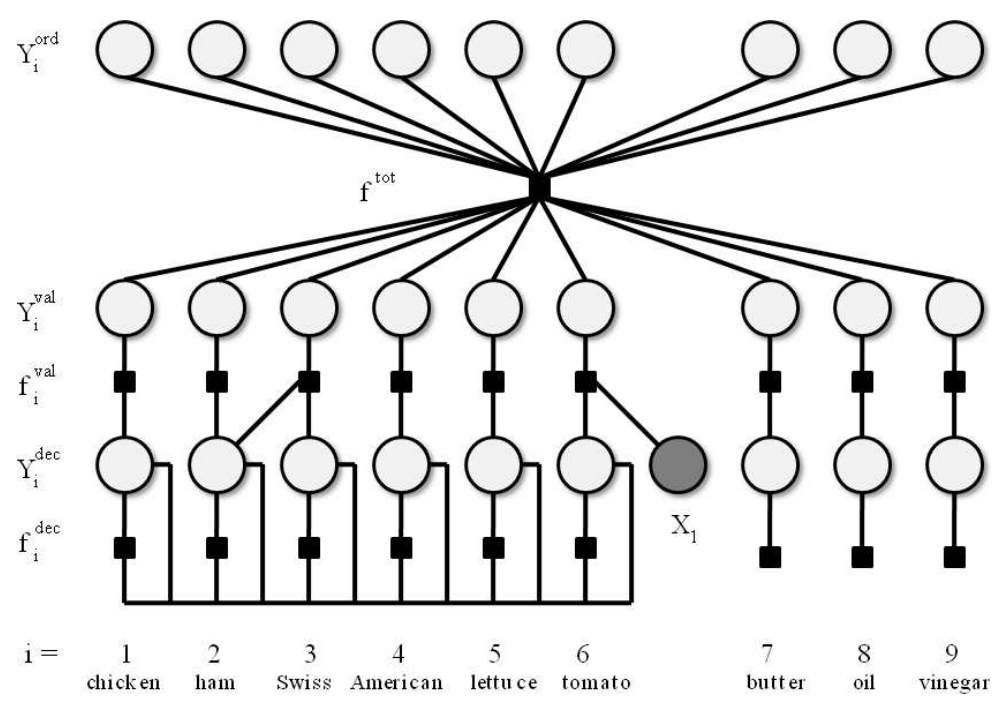

Figure 2: Example sandwich-making DARN model (graphical level)

However, it is first necessary to describe the general message passing process used for DARN inference. Since $p(Y \mid$ $X)=u(\cdot)$, an optimal strategy is where the CRF variable encoding, $Y_{\text {optimal }}$, is such that $Y_{\text {optimal }}=\operatorname{argmax}_{Y} p(Y \mid X)$. This probability may be approximated by message accumulation, as follows:

$$
\begin{aligned}
& m_{V \rightarrow F}^{(1)}(x)=1 \\
& m_{V \rightarrow F}^{(k)}(x)=\prod_{F^{\prime} \in N(V) \backslash F} m_{F^{\prime} \rightarrow V}^{(k-1)}(x) \\
& m_{F \rightarrow V}^{(k)}(x)=\sum_{X^{\prime}: x \notin X^{\prime}} \exp \left(F\left(x, X^{\prime}\right)\right) \prod_{V^{\prime} \in N(F) \backslash V} m_{V^{\prime} \rightarrow F}^{(k-1)}(x) \\
& p^{(k)}(x)=\sum_{X^{\prime}: x \notin X^{\prime}} \exp \left(f^{t o t}\left(x, X^{\prime}\right)\right) \prod_{V^{\prime} \in N\left(f^{t o t}\right)} m_{V^{\prime} \rightarrow f^{t o t}}^{(k-1)}(x)
\end{aligned}
$$

where:

- $k$ is the number of an arbitrary iteration

- $x$ is one or more variable assignments; messages with no trailing $x$ cover all assignments

- $m_{A \rightarrow B}^{(k)}(\cdot)$ is the message from graph node $A$ to graph node $B$ during iteration $k$ (regarding the parameters)

- $F$ and $F^{\prime}$ are arbitrary factor nodes

- $V$ and $V^{\prime}$ are arbitrary variable nodes

- $N(\cdot)$ is a function that returns the set of all adjacent graph nodes

- $\sum_{X^{\prime}: x \notin X^{\prime}}$ refers to the summation over all parameter assignments excluding $x$

- $F(\cdot)$ is the computation of the function whose factor node is $F$ (ignoring $x$ if it is not in the parameter list of $F(\cdot)$ )

The desire is to compute $p^{(k)}(Y \mid X)$ (for some $k$ ) where $p^{(k)}(Y \mid X)=p(Y \mid X)\left(\right.$ or $\left|p^{(k)}(Y, X)-p(Y \mid X)\right|<\epsilon$ for some small $\epsilon$, when approximating). Messages propagating from the $f_{i}^{d e c}(\cdot)$ and $f_{i}^{v a l}(\cdot)$ nodes indicate variable assignment constraints that must be taken into account during final valuation. Exact inference will include the propagation of all of these messages, while inexact (approximate) inference will propagate a growing proportion.

The impact of message ordering on general loopy CRFs is still poorly understood, although recent publications have suggested approaches aimed at reducing the number of messages without compromising precision. For instance, [Sutton et al., 2007] support the adaptation of Tree Reparameterization (TRP) for general CRFs, where messages are generated along cross-cutting spanning trees and tree selection is performed randomly. Although TRP is expected to perform well at propagating decision ordering information and generally passing information between local trees in a DARN model, the introduction of cross-decision restrictions or valuations (from the parametrization of $Y_{i}^{d e c}$ and $Y_{i}^{v a l}$ ) suggests a large expected number of iterations required to converge if using random tree selection. Using TRP as our motivation, we propose message ordering schemes designed to use small spanning trees for message propagation while using custom tree orderings that outperform random selection.

We begin with an observation: although it is not possible to reduce the number of computations of $f_{i}^{\text {dec }}(\cdot)$, it is possible to reduce the number of computations of $f_{i}^{v a l}(\cdot)$ and $f^{t o t}(\cdot)$ by ensuring that messages sent outwards from those nodes follow certain incoming messages. Specifically, if $m_{f_{i}^{\text {val }} \rightarrow V}^{(k)}(x)$ is not computed before $m_{f_{i}^{\text {dec }} \rightarrow Y_{i}^{\text {dec }}}^{(j)}(x)$ and $m_{Y_{i}^{\text {dec }} \rightarrow f_{i}^{\text {val }}}^{\left(j^{\prime}\right)}(x)$ (for $j<j^{\prime}<k$ ), then it is possible to register 0 -valued entries for assignments in $m_{f_{i}^{\text {val }} \rightarrow V}^{(k)}(x)$ where $f_{i}^{\text {dec }}(\cdot)=-\infty$ without computing $f_{i}^{v a l}(\cdot)$. So, it is ideal to pass messages among decision-local nodes before local valuation nodes, and local valuation nodes before the global valuation node, to reduce the number of functions computed for disallowed decisions. 
However, it is still possible that valuations will be calculated for sets of decisions that are not allowed, as there may be situations where setting one decision adds constraints upon another decision; these can be eliminated if $m_{f_{i}^{d e c} \rightarrow V}^{(k)}$ propagate prior to further calculation. These two notions give us the exact message passing scheme shown as Algorithm 1.

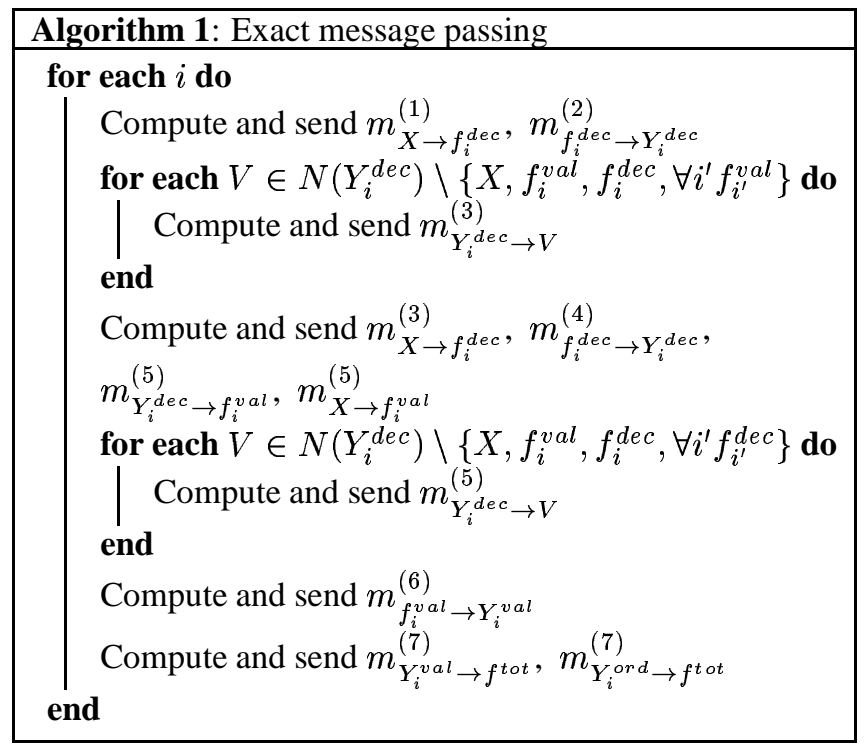

The result of Algorithm 1 is to produce a set of messages such that $p^{(8)}(Y, X)=p(Y \mid X)$ using a near-optimal number of function computations; improvement is only possible when the algorithm is tailored to the specific constraints present, and can require overwhelming computational cost to determine an optimal ordering as the number of decisions and connections increases. It is important to note that messages sent more than once (e.g, from $X$ to $f_{i}^{d e c}$ ) may be stored without recomputing, and that modifications to these messages (due to other incoming messages) is performed by zeroing existing entries without recomputing any factor functions. This "storing" may be applied to future inference performed on the same DARN model but with partially-varying $X$, thereby allowing for a reduction in the number of computations when only a portion of the decision space has been changed, even to the point where no additional functions need be calculated. This property may be viewed as "training" by storing valuations on given data, then using these valuations to perform fast inference on test data (by substituting training valuations for approximately-equivalent test situations while attempting to minimize computational error). We will explore this property in greater depth in future publications, especially as it pertains to inference in large DARN models for Computer Vision tasks.

An approximate version of Algorithm 1 is provided in Algorithm 2, with the additional requirements that:

- $m_{A \rightarrow B}^{(0)}(x)=0$ for all nodes A and B where $A \notin X$

- $m_{A \rightarrow B}^{(j+1)}(x)=m_{A \rightarrow B}^{(j)}(x)(\forall j \geq 0)$ unless specified

- $\hat{i}$ is the set of all values not chosen from $i$ so far
- If $\hat{i}=\emptyset$ at the end of any iteration, $\hat{i}=i$ at the start of the next iteration

These properties ensure that the set of valuated assignments is initialized to be empty and grows as $k$ increases past $m$. This may incorrectly assume high-valued assignments to be disallowed, but will converge exactly as $k$ approaches $2 m$ (where worst-case selection will require $2 m$ iterations for all inter-decision messages). The rate of convergence is dependent upon the structure of the modeled problem and the sampling schema used for each iteration; we suggest an initial scheme where the selection from $\hat{i}$ maximizes the number of $m_{V \rightarrow F}^{(k)}$ messages sent during each iteration.

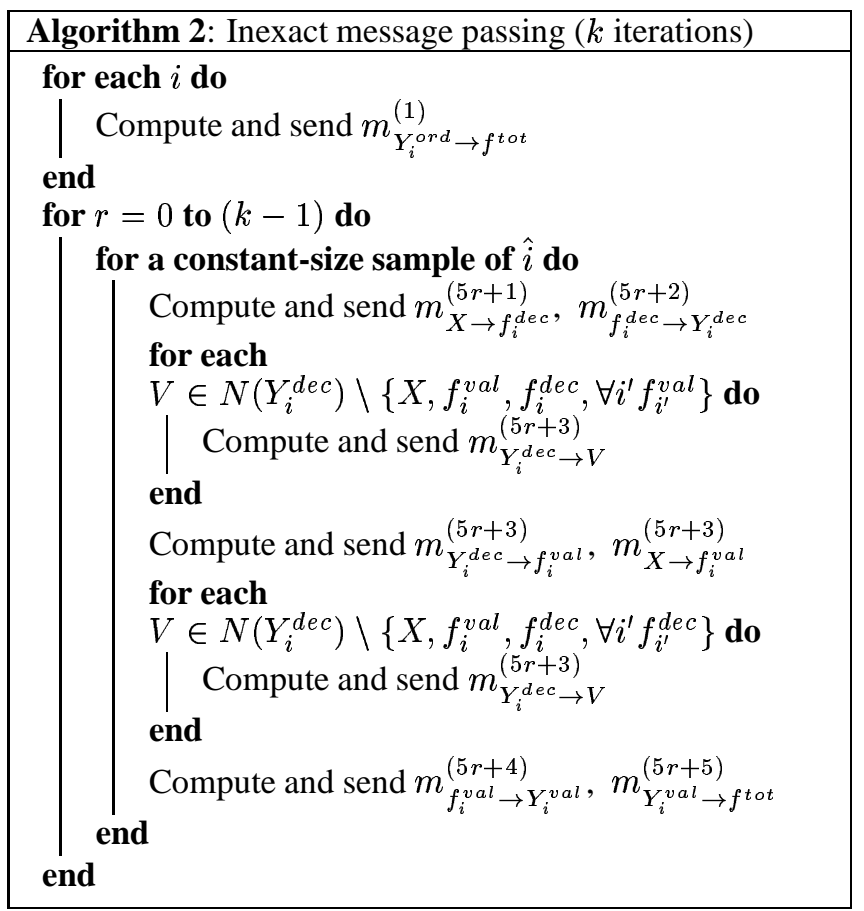

Once $p(Y \mid X)$ is computed or approximated for all feasible decisions, and given $X$, inference terminates by selecting a setting of $Y$ with highest conditional probability. If there are multiple such settings, selection is arbitrary. Algorithm 2 must be performed for a larger value of $k$ if no values for $Y$ are approximated as having non-zero conditional probability. Cases where there are no non-zero valuations (i.e., where approximation will never terminate) are treated as degenerate.

Using this inference mechanism, it is now possible to compute an optimal sandwich construction, albeit only if tomato quality is already known. We will next show how to take this "tomato uncertainty" into account.

\section{Solving General Problems}

To convert the instance-specific framework into one that will solve the general decision problem, we need only introduce information about $\mathbf{C}$ into the existing graphical structure. Specifically, we convert the CRF to a Markov Random Field (MRF) [Kindermann and Snell, 1980], adding a new factor function $f^{p r o b}(X)=p(X)$. Graphically, this factor node is 
connected to all (now unobserved) $X_{i}$ nodes; if $X$ contains independent subsets, each subset may have a disparate new factor node that is connected only to its corresponding $X_{i}$ nodes, returning the joint probability of the elements of the subset. The message-passing computation with these additional factors (introducing $m_{f^{p r o b} \rightarrow X_{i}}^{0}$ messages and replacing constant $m_{X_{i} \rightarrow V}^{1}$ with computed $m_{X_{i} \rightarrow V}^{k}$ for $k=1$ ) results in the joint probability of $Y$ and $X$, so an optimal strategy can be determined by selecting assignments to $Y$ that maximize this probability; this is equivalent to finding $\operatorname{argmax}_{Y} \mathrm{E}[u(\cdot)]_{X} \cdot$

Returning to the sandwich-making example, we set $f^{\text {prob }}=0.5$, so the expected utility of the ham-and-Swiss sandwich (22) is strictly greater than the ham-Swiss-tomato sandwich (21) as anticipated. Thus, it is best to avoid tomatoes if quality is unknown. We have also applied this approach to Towers of Hanoi and chess "opening book" planning, but omit these results due to space constraints.

Although it is possible to produce alternate graphical models that compute this result without relying on existing (incompact) tools, we prefer the use of CRFs (and MRFs). Of particular interest, given recent trends, is the possibility of using an MLN to encode the hard constraints of $g_{i}(\cdot)$ computations as well as the comparable utility score. While it is provably feasible to produce an MLN that embodies the same probability distribution as our CRF-based approach (as MLNs subsume all discrete graphical models), it is not possible to produce one with comparable compactness. The reason for this lies in the encoding of the distribution such that the ordering of probabilities of variable assignments (groundings) preserves the ordering of the full utility valuation. For this, the probability of a particular ground MLN (where all variables have received assignments) must appears in the same order relative to all other ground MLNs as its utility valuation does to all other feasible valuations. As the probability of a particular ground MLN is equal to the exponentiation of summed weighted formula truths, this means that an MLN that preserves valuation ordering must have formula weights that produce an equivalent ordering. However, according to the definition of an MLN, "all groundings of the same formula will have the same weight". To produce correctlyordered ground probabilities, therefore, the MLN must include disparate formulas for each possible utility valuation (with according weight and limited satisfiability), resulting in an exponential growth in the number of graph nodes.

\section{Conclusions}

Overall, the proposed DARN model is an effective tool for modeling decisions where ordering may or may not be constrained and where valuation is not necessarily additive or multiplicative. We suggest efficient algorithms for exact and inexact inference; future work will study more sophisticated guidance during inexact inference and the "training" mechanism suggested in Section 4.

\section{Acknowledgements}

Special thanks to Chris Pal for his discussions, and to the late Henry E. Kyburg, Jr. for his rules of sandwich-making.

\section{References}

[Demirer and Shenoy, 2006] Riza Demirer and Prakash P. Shenoy. Sequential valuations networks for asymmetric decision problems. European Journal of Operational Research, 169(1):286-309, 2006.

[Jensen and Vomlelova, 2002] F. V. Jensen and M. Vomlelova. Unconstrained influence diagrams. In Proceedings of the 18th Conference on Uncertainty in Artificial Intelligence, pages 234-241, 2002.

[Kearns et al., 1999] M. Kearns, Y. Mansour, and A. Y. Ng. A sparse sampling algorithm for near-optimal planning in large markov decision processes. In Proceedings of the International Joint Conference on Artificial Intelligence 1999, pages 1324-1331, 1999.

[Kindermann and Snell, 1980] Ross Kindermann and J. Laurie Snell. Markov Random Fields and their Applications. American Mathematical Society, 1980.

[Kschischang et al., 2001] F. R. Kschischang, B. J. Frey, and H. A. Loeliger. Factor graphs and the sum-product algorithm. IEEE Transactions on Information Theory, 47(2):498-519, 2001.

[Lafferty and Pereira, 2001] John Lafferty and Fernando Pereira. Conditional random fields: Probabilistic models for segmenting and labeling sequence data. In Proceedings of the 18th International Conference on Machine Learning, pages 282-289, 2001.

[Pal et al., 2006] Chris Pal, Charles Sutton, and Andrew McCallum. Sparse forward-backward using minimum divergence beams for fast training of conditional random fields. In Proceedings of the 2006 International Conference on Acoustics, Speech, and Signal Processing, volume 5, pages 581-584, 2006.

[Pearl, 1988] J. Pearl. Probabilistic Reasoning in Intelligent Systems: Networks of Plausible Inference. Morgan Kaufmann, 1988.

[Richardson and Domingos, 2006] Matthew Richardson and Pedro Domingos. Markov logic networks. Machine Learning, 62:107-136, 2006.

[Shenoy, 2000] Prakash P. Shenoy. Valuation network representation and solution of asymmetric decision problems. European Journal of Operational Research, 121(3):579_ 608, 2000.

[Sutton et al., 2007] Charles Sutton, Andrew McCallum, and Khashayar Rohanimanesh. Dynamic conditional random fields: Factorized probabilistic models for labeling and segmenting sequential data. The Journal of Machine Learning Research, 8:693-723, 2007.

[Weinman et al., 2008] Jerod Weinman, Lam Tran, and Chris Pal. Efficiently learning random fields for stereo vision with sparse message passing. In Proceedings of the Eleventh European Conference on Computer Vision, 2008.

[Yedidia et al., 2001] Jonathan S. Yedidia, William T. Freeman, and Yair Weiss. Generalized belief propagation. In Neural Information Processing Systems, pages 689-695, 2001. 